EPJ Web of Conferences 72, 00018 (2014)

DOI: 10.1051/epjconf / 20147200018

(C) Owned by the authors, published by EDP Sciences, 2014

\title{
Recent Progress in Tau Lepton Physics
}

\author{
Ian M. Nugent ${ }^{1,3, a}$ \\ ${ }^{1}$ III. Physikalisches Institut \\ Physikzentrum \\ RWTH Aachen \\ 52056 Aachen, Germany
}

\begin{abstract}
The $\tau$ lepton is the heaviest of the charged leptons and the only one to decay into hadrons. This provides a unique opportunity to probe low energy QCD and the charged weak interaction of both the relative coupling strength to the first and second generation of quarks and to the lepton. Moreover, many new physics scenarios couple primarily to the third generation, making it a promising prospect for new physics searches. In this paper, an overview of recent measurements of the $\tau$ lepton from BABAR, BELLE and BES are presented.
\end{abstract}

\section{Introduction}

$\tau$ decays at low energy $e^{+} e^{-}$colliders provide an experimentally clean signature to studying electro-weak interactions, low energy QCD and for searching for new physics. The $\tau$ lepton is of particular interest for new physics searches due to its mass and the flavour problem. At low energies, new physics can manifest itself through virtual contributions from new particles. Two of the key signatures searched for at the B-Factories are Lepton Flavour Violation and CP Violation. With the large datasets collected by the BELLE and BABAR experiments there is a unique opportunity to probe the Standard Model by using the rich dynamics of $\tau$ decays to an unprecedented precision. Furthermore, the enormous statistics are essential for the searches for new physics.

From the B-Factories, there have been many measurements of branching fractions/ratios from $\tau$ decay modes, as well as numerous searches for new decay modes [1]. This includes the majority of the main strange $\tau$ branching fractions and upper limits on the unmeasured strange decay modes[2, 3]. Table 1 presents a summary of the non-strange and strange branching fraction measurements as well as the upper limits on unmeasured strange decay from the BELLE and BABAR experiments. As it is pointed out in [1], the measured decay modes from BABAR and BELLE tend to be lower than previous experiments. However, it should be pointed out although the new sum of branching fractions is low, it is still reasonably consistent with unity [1].

${ }^{\mathrm{a}}$ I would like to thank conference organizers, F. Curciarello, V. De Leo, G. Fazio , G. Giardina, G. Mandaglio and F. Mammoliti, and S. Eidelman for giving me this opportunity to present recent results on $\tau$ decays. Personal support for Ian M. Nugent was provided by the Alexander Von Humboldt Foundation. e-mail: nugent@physik.rwth-aachen.de
In Section 2 and 3, the searches for new physics through Lepton Flavour Violation and CP Violation will be discussed. Search for Second Class Currents will be discussed in Section 4. After which, the precision measurements of the Standard Model, at low energy $e^{+} e^{-}$colliders, will be discussed. These include the measurements of the $\tau$ mass, which are presented in Section 5, and the electro-weak measurements, which are presented in Section 6. The discussion of the hadronic structure and QCD parameters is left to Section 7. Finally, in Section 8 an outlook for $\tau$ physics is presented.

\section{Searches for Lepton Flavour Violation}

Lepton Flavour Violation (LFV) is forbidden in the Standard Model of Particle Physics and is strongly suppressed when neutrino oscillations are taken into account. However, there are many new physics scenarios that predict LFV could occur at the $O\left(10^{-8}\right)$ level[17-24]. Any observation of LFV would be a clear signature of new physics. Because of this, LFV was intensively searched for at the BABAR and BELLE experiments[1]. The current world limit, as summarized by the heavy Flavour Averaging Group (HFAG), are presented in Figure 1. In addition to LFV, these searches also include Lepton Number Violation and Baryon Number Violation. With the expected luminosity at the BELLE II Experiment, LFV searches promise to be an exciting topic for $\tau$ physics.

\section{Searches for CP Violation in $\tau$ Decays}

In the Standard Model, CP Violation results from the single irreducible complex phase in the Cabibbo-KobayashiMaskawa matrix (CKM) [25]. It explains all CP Violation effects that have been measured, which so far are limited to 
EPJ Web of Conferences

Table 1. The HFAG fit branching fraction for both strange and non-strange $\tau$ decays which were measured by the B-Factories [1]. A more complete list of decay modes measured by BABAR and BELLE can be found in [1].

\begin{tabular}{|c|c|c|c|}
\hline Decay Mode & Branching Fraction (\%) & BELLE & $B A B A R$ \\
\hline $\mathcal{B}(\tau \rightarrow e v \bar{v})$ & $17.82 \pm 0.04$ & & [5] \\
\hline $\mathcal{B}(\tau \rightarrow \mu \nu \bar{v})$ & $17.39 \pm 0.04$ & & [5] \\
\hline $\mathcal{B}(\tau \rightarrow \pi \nu)$ & $10.81 \pm 0.05$ & & [5] \\
\hline $\mathcal{B}\left(\tau^{-} \rightarrow h^{-} \pi^{0} \nu_{\tau}\right)$ & $25.94 \pm 0.09$ & [6] & \\
\hline $\mathcal{B}\left(\tau^{-} \rightarrow \pi^{-} \pi^{-} \pi^{+} v_{\tau}\left[\right.\right.$ ex. $\left.\left.K_{S}^{0}\right]\right)$ & $9.00 \pm 0.05$ & [7] & [8] \\
\hline $\mathcal{B}\left(\tau^{-} \rightarrow \pi^{-} \pi^{0} \eta v_{\tau}\right)$ & $0.139 \pm 0.007$ & [9] & \\
\hline $\mathcal{B}\left(\tau^{-} \rightarrow 3 \pi^{-} 2 \pi^{+} v_{\tau}\left[\right.\right.$ ex. $\left.\left.K_{S}^{0}\right]\right)$ & $0.0823 \pm 0.0030$ & & [10] \\
\hline $\mathcal{B}\left(\tau^{-} \rightarrow 2 \pi^{-} \pi^{+} \eta \nu_{\tau}\right)$ & $0.0149 \pm 0.0010$ & & [11] \\
\hline $\mathcal{B}\left(\tau^{-} \rightarrow K^{-} \pi^{-} \pi^{+} \nu_{\tau}\right)$ & $0.1435 \pm 0.0027$ & [7] & [8] \\
\hline $\mathcal{B}\left(\tau^{-} \rightarrow \pi^{-} K_{S}^{0} \pi^{0} v_{\tau}\right)$ & $0.145 \pm 0.007$ & [12] & \\
\hline $\mathcal{B}(\tau \rightarrow K \nu)$ & $0.6955 \pm 0.0096$ & & [5] \\
\hline $\mathcal{B}\left(\tau^{-} \rightarrow K^{-} \pi^{0} v_{\tau}\right)$ & $0.4322 \pm 0.0149$ & & [13] \\
\hline $\mathcal{B}\left(\tau^{-} \rightarrow K^{0} \pi^{-} v_{\tau}\right)$ & $0.8206 \pm 0.0182$ & [6] & [14] \\
\hline $\mathcal{B}\left(\tau^{-} \rightarrow K^{0} \pi^{-} \pi^{0} v_{\tau}\right)$ & $0.3649 \pm 0.0108$ & [12] & [15] \\
\hline $\mathcal{B}\left(\tau^{-} \rightarrow K^{-} \pi^{-} \pi^{+} \nu_{\tau}\left(\right.\right.$ ex. $\left.\left.K^{0}\right)\right)$ & $0.2923 \pm 0.0068$ & [7] & [8] \\
\hline $\mathcal{B}\left(\tau^{-} \rightarrow K^{-} \eta \nu_{\tau}\right)$ & $0.0153 \pm 0.0008$ & [9] & [16] \\
\hline $\mathcal{B}\left(\tau^{-} \rightarrow K^{-} \eta \pi^{0} \nu_{\tau}\right)$ & $0.0048 \pm 0.0012$ & [9] & \\
\hline $\mathcal{B}\left(\tau^{-} \rightarrow K^{0} \eta \pi^{-} \nu_{\tau}\right)$ & $0.0094 \pm 0.0015$ & [9] & \\
\hline $\mathcal{B}\left(\tau^{-} \rightarrow K^{-} \phi v_{\tau}\left(\phi \rightarrow K^{-} K^{+}\right)\right)$ & $0.0037 \pm 0.0014$ & [7] & [8] \\
\hline
\end{tabular}

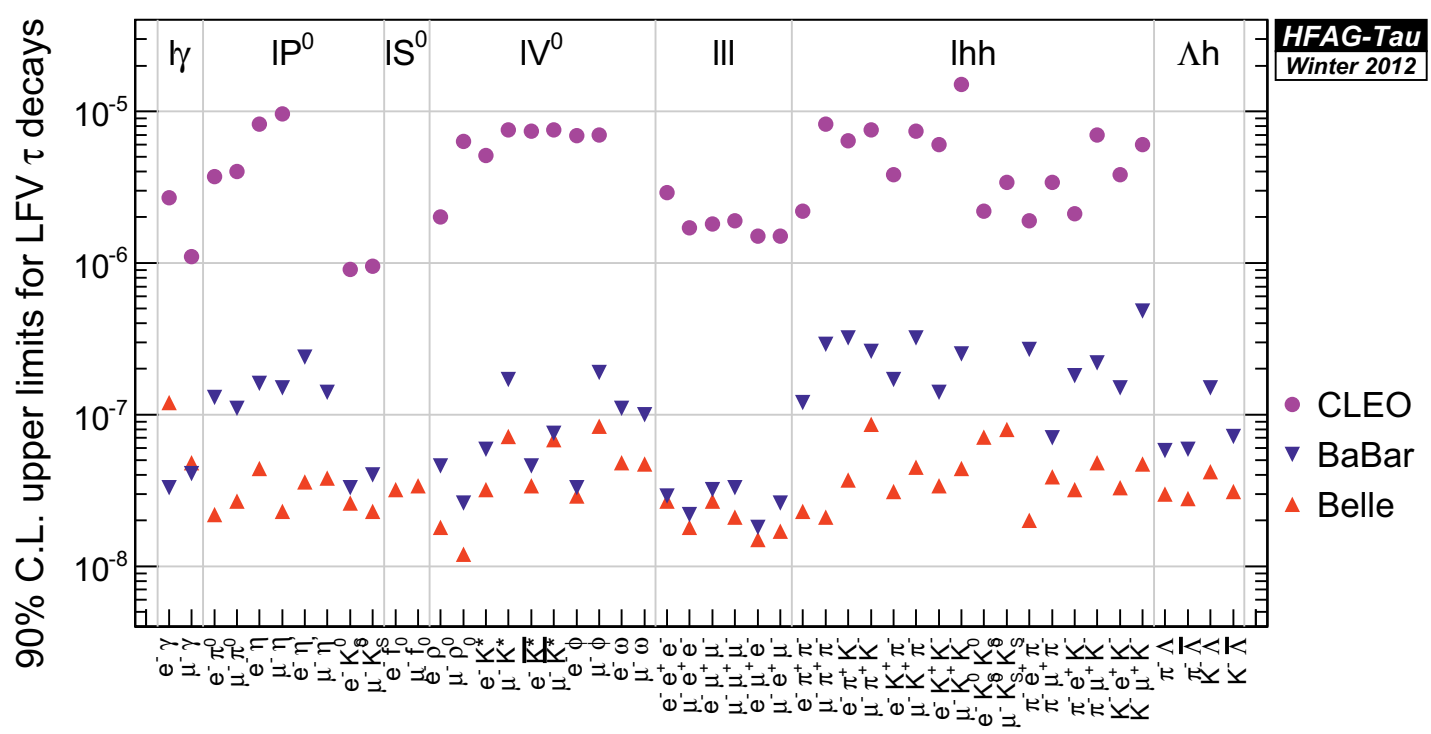

Figure 1. A summary of the LFV from BELLE and BABAR as presented in the HFAG 2012 report[1]

the $\mathrm{K}$ and $\mathrm{B}$ mesons. However, the amount of $\mathrm{CP}$ Violation predicted by the Standard Model is several orders of magnitude too small to explain the asymmetry between matter and antimatter in the universe. Thus searching for sources of new CP Violation is essential to answer this fundamental question in physics. Many new physics models predict new sources of CP Violation, for example from scalar bosons in $\tau$ decays which are expected in models such as Minimal Supersymmetric Standard Models[26, 27] and from Multi-Higgs-Doublet Models[28, 29]. This was the focus of the CP Violation search at BELLE [30] which measured differential CP asymmetry,

$$
\begin{aligned}
A_{i}^{C P} & =\frac{\iiint_{Q_{1, i}^{2}}^{Q_{2, i}^{2}} \cos \beta \cos \psi\left(\frac{d \Gamma_{\tau^{-}}}{d \omega}-\frac{d \Gamma_{\tau^{+}}}{d \omega}\right) d \omega}{\frac{1}{2} \iiint_{Q_{1, i}^{2}}^{Q_{2, i}^{2}}\left(\frac{d \Gamma_{\tau^{-}}}{d \omega}-\frac{d \Gamma_{\tau^{+}}}{d \omega}\right)} \\
& \simeq\langle\cos \beta \cos \psi\rangle_{\tau^{-}}^{i}-\langle\cos \beta \cos \psi\rangle_{\tau^{+}}^{i},
\end{aligned}
$$

where $d \omega=d Q^{2} d \cos \beta d \cos \theta$ using the angular definitions from [31]. The results of this search can be seen in Figure 2. In the context of new physics models, where the scalar form factor is modified by the Charged Higgs contributions

$$
F_{S}\left(Q^{2}\right) \rightarrow F_{S}^{\prime}\left(Q^{2}\right)=F_{S}\left(Q^{2}\right)+\frac{\eta_{S}}{m_{\tau}} F_{H}\left(Q^{2}\right)
$$



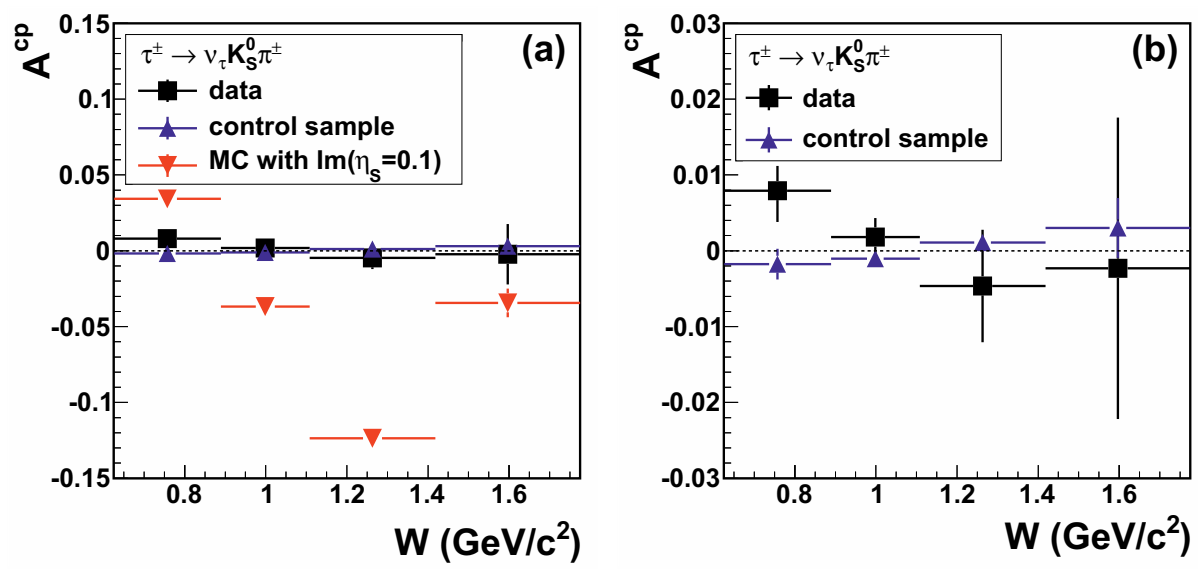

Figure 2. The CP asymmetry, $A_{i}^{C P}$, measured by BELLE[30, Figure 2]. The vertical bars represent the statistical and systematic uncertainties added in quadrature for Data. For the control sample, the vertical error bars represent the statistical uncertainty. The difference between the left-hand plot (a) and the right-hand plot (b) is the scale of $A_{i}^{C P}$.

$A_{i}^{C P}$ is proportional to the imaginary component of the dimensionless complex coupling constant[30]. The resulting upper limit on the new physics models is $\left|\mathfrak{J} m\left(\eta_{S}\right)\right|<$ 0.026@90\%CL. For Multi-Higgs-Doublet Models[28, 29], one can place a limit on the complex coupling constants of the Higgs boson to $\tau-v_{\tau}(\mathrm{Z})$ and the $u-d$ quarks (Z) $[30,32]$. The resulting upper limit from BELLE[30] is

$$
\left|\mathfrak{J} m\left(X Z^{*}\right)\right|<0.15 \frac{M_{H^{ \pm}}^{2}}{1 G e V^{2} / c^{4}} @ 90 \% C L .
$$

The BABAR experiment conducted a search for a global CP asymmetry which measured an asymmetry of $(-0.36 \pm$ $0.23 \pm 0.11) \%$ [33]. This is $2.8 \sigma$ away from the Standard Model prediction of $(0.36 \pm 0.01) \%$.

\section{Searches for Second Class Currents}

Hadronic currents in weak interactions can be categorized in two general groups based on the spin, parity and $\mathrm{G}$ parity[4]. First Class Currents, FCC, have a $J^{P G}=$ $0^{++}, 0^{--}, 1^{+-}, 1^{-+}$, while the Second Class Currents, SCC, have a $J^{P G}=0^{-+}, 0^{-+}, 1^{++}, 1^{--}$. The SCC correspond to matrix elements that are proportional to the difference between the up and down quark masses and are therefore highly suppressed. They have never been observed before. SCC are predicted to be on the order of $(1.2-1.5)^{-5}$ $[34,35]$. At the B-Factories, SCC have been searched for in the $\tau^{-} \rightarrow \pi^{-} \eta v_{\tau}[16], \tau^{-} \rightarrow \pi^{-} \eta^{\prime} v_{\tau}[11]$ and the $\tau^{-} \rightarrow \pi^{-} \omega v_{\tau}[36]$ decay modes. A summary of the results can be found in Table 2 .

Within the Resonance Chiral Lagrangian Models, $\operatorname{Br}\left(\tau^{-} \rightarrow \pi^{-} \eta \nu_{\tau}\right)$ is predicted to be $\left(0.48_{-0.20}^{+0.30}\right) \times 10^{-5}[37]$, about one order of magnitude below the current experimental measurements. This is near the limit of the expected reach of the BELLE II Experiment for the full expected luminosity.
Table 2. Upper limits set on Second Class Currents at the B-Factories

\begin{tabular}{lrr}
\hline \hline Decay Mode & Upper Limit (90\% CL) & Experiment \\
$\tau^{-} \rightarrow \pi^{-} \eta v_{\tau}$ & $<9.9 \times 10^{-5} @ 90 \% C L$ & BABAR [16] \\
$\tau^{-} \rightarrow \pi^{-} \eta v_{\tau}$ & $<7.3 \times 10^{-5} @ 90 \% C L$ & BELLE \\
$\tau^{-} \rightarrow \pi^{-} \eta^{\prime} v_{\tau}$ & $<7.2 \times 10^{-6} @ 90 \% C L$ & BABAR [11] \\
$\tau^{-} \rightarrow \pi^{-} \eta^{\prime} v_{\tau}$ & $<4.6 \times 10^{-6} @ 90 \% C L$ & BELLE \\
$\tau^{-} \rightarrow \pi^{-} \omega v_{\tau}$ & $<1.3 \times 10^{-4} @ 90 \% C L$ & BABAR [36] \\
\hline \hline
\end{tabular}

\section{Measurements of the $\tau$ Mass}

The $\tau$ mass is one of the fundamental parameters of the Standard Model. As such, it can not be predicted by theory but can only be measured. Precision measurements of the $\tau$ mass allow for both calculating branching fractions[38] and for testing Charged Lepton Universality[39]. While the differences between the $\tau^{-}$and $\tau^{+}$masses provide a test of CPT, any violation of CPT would imply violation of local Lorentz invariance and would indicate new physics[40-43]. The most precise measurements of the $\tau$ mass come from threshold scans. In particular, the most precise measurements of the $\tau$ mass using this technique come from KEDR (2009), 1776.69 $+0.17 \pm 0.15 \mathrm{MeV} / \mathrm{c}^{2}$ [44], and BES III(2013) $1776.91 \pm 0.12 \pm 0.12 \mathrm{MeV} / \mathrm{c}^{2}[45]$. Complementary measurements of differences between the $\tau^{-}$and $\tau^{+}$masses have been made at BABAR $(1776.68 \pm$ $\left.0.12 \pm 0.41 \mathrm{MeV} / \mathrm{c}^{2}\right)[46]$ and BELLE $(1776.61 \pm 0.13 \pm$ $0.35 \mathrm{MeV}$ )[47] using the pseudo-mass technique.

\section{Measurements of the Electro-weak Couplings}

In the Standard Model, the interaction of the charged weak current with the quarks is described with a single gauge coupling and a unitary transform matrix $\left(\left|V_{u s}\right|\right)$, the Cabibbo-Kobayashi-Maskawa matrix (CKM) [25], which 
results from the mass and weak eigenstates of the quarks not being equivalent:

$$
-\frac{g}{\sqrt{2}}\left[\bar{u}_{i} \gamma^{\mu} \frac{1-\gamma^{5}}{2} V_{i j} d_{j}+\bar{v}_{i} \gamma^{\mu} \frac{1-\gamma^{5}}{2} l_{i}\right] W_{\mu}^{+}
$$

+higher order terms,

where $g=g_{f}=g_{e}=g_{\mu}=g_{\tau}$ is the signal gauge coupling, $u_{i}$ and $d_{i}$ represent the up-down-like quark states, $l_{i}$ and $v_{i}$ represent the charged lepton and neutrino states of the $i$ th generation and $\frac{1-\gamma^{5}}{2}$ forces the interaction to be left handed. The equality between the gauge couplings for the three generations of leptons is referred to as Charged Lepton Universality. For the first row of the CKM matrix, the unitarity constraint may be written as

$$
V_{u d}+V_{u s}+V_{u b}=1,
$$

where the value of $\left|V_{u d}\right|$ used in this comparison is provided from nuclear beta decays [48], the contribution from $\left|V_{u b}\right|$ is negligible[1] and $\left|V_{u s}\right|$ comes from kaon decay measurements[49]. However, hadronic $\tau$ decays provide a complementary method for extracting $\left|V_{u s}\right|$. This is of particular interest since there are many new physics scenarios that couple primarily to the third generation and could cause a deviation between measurements of $\left|V_{u s}\right|$ in the kaon and $\tau$ systems[50-54].

\section{1 $\left|V_{u s}\right|$ in $\tau$ Decays}

In $\tau$ decays, there are four main techniques that can be used to extract $\left|V_{u s}\right|$. In this paper we will discuss the three methods that have been used at the B-Factories. These techniques only require input from $\tau$ decays. A description of the fourth method which combines measurements from $\tau \rightarrow K \pi v$ decays and $K \rightarrow \pi l v$ decays can be found in [55]. Out of the $\tau$ only techniques, the method that has the smallest theoretical limitations comes from the flavor breaking difference with Finite Energy Sum Rules (FESR)[56]. The flavor breaking difference can be written as

$$
\frac{R_{\tau, \text { strange }}^{w}}{\left|V_{u s}\right|^{2}}-\frac{R_{\tau, \text { non-strange }}^{w}}{\left|V_{u d}\right|^{2}}=\delta R_{\tau, S U 3 \text { breaking }}^{w},
$$

where $R_{\tau, \text { strange }}=\Gamma\left(\tau^{-} \rightarrow X_{\text {strange }} \nu_{\tau}\right) / \Gamma(\tau \rightarrow e v \bar{v})$ is the strange hadronic width, $R_{\tau, \text { non-strange }}=\Gamma\left(\tau^{-} \rightarrow\right.$ $\left.X_{\text {non-strange }} v_{\tau}\right) / \Gamma(\tau \rightarrow e v \bar{v})$ is the non-strange hadronic width and $\delta R_{\tau, S U 3}$ breaking is the theoretical SU(3) flavor breaking correction which is determined using Operator Product Expansion (OPE). The $w$ represents that this equation holds for any analytic weight. In general, the weights can be constructed to minimize the total uncertainty. However, due to the limited amount of information on the inclusive spectral density functions of the current experimental measurements, the results presented here are unweighted. The weighted inclusive strange and non-strange spectral density functions are constructed from the desired analytic weight, the sum of invariant mass distributions for each of the strange and non-strange decay modes and normalized to the corresponding branching fractions. Experimentally, this is challenging due to the number of channels that need to be measured. Moreover, since there are no solid predictions for the branching fractions of hadronic individual $\tau$ decays, all possible modes must be measured or have an upper bound placed on them. This technique has completely orthogonal theoretical and experimental uncertainties to the kaon measurements. If all of the branching fractions and spectral functions are updated with the data from BELLE and BABAR, this method would be expected to make the most precise measurement of $\left|V_{u s}\right|$ [56].

The most precise technique for determining $\left|V_{u s}\right|$ from $\tau$ decays is currently from the branching ratio:

$$
\frac{\mathcal{B}(\tau \rightarrow K v)}{\mathcal{B}(\tau \rightarrow \pi v)}=\frac{f_{K}^{2}\left|V_{u s}\right|^{2}}{f_{\pi}^{2}\left|V_{u d}\right|^{2}} \frac{\left(1-\frac{m_{K}^{2}}{m_{\tau}^{2}}\right)^{2}}{\left(1-\frac{m_{\pi}^{2}}{m_{\tau}^{2}}\right)^{2}}\left(1+\delta_{L D}\right),
$$

where $f_{K} / f_{\pi}=1.1936 \pm 0.0053$ [57-60] is determined from Lattice QCD, $\left|V_{u d}\right|$ [48], and the long-distance correction $\delta_{L D}=(0.03 \pm 0.44) \%$ is estimated [61] using corrections to $\tau \rightarrow h v_{\tau}$ and $h \rightarrow \mu v_{\mu}[62,63]$. This method is analogous to the method used in $K_{l 2}$ decays and therefore has the same Lattice QCD uncertainties.

$\left|V_{u s}\right|$ can be directly extracted from the measurement of the branching fraction $\tau^{-} \rightarrow K^{-} v_{\tau}$,

$$
\mathcal{B}(\tau \rightarrow K v)=\frac{G_{F}^{2} f_{K}^{2}\left|V_{u s}\right|^{2} m_{\tau}^{3} \tau_{\tau}}{16 \pi \hbar}\left(1-\frac{m_{K}^{2}}{m_{\tau}^{2}}\right)^{2} S_{E W},
$$

where the kaon decay constant is $f_{K}=156.1 \pm$ $1.1 \mathrm{MeV}$ [57-60] and the electro-weak correction is $S_{E W}=1.0201 \pm 0.0003$ [64]. In contrast to the latter method, this method is sensitive to the absolute normalization, however, by construction it is also affected by Lattice QCD uncertainties.

The extraction of $\left|V_{u s}\right|$ from the B-Factories results is computed by HFAG using the fitted HFAG branching fractions. The HFAG values of $\left|V_{u s}\right|$ compared to the kaon measurements are presented in Figure 3. In contrast to the kaon measurements, the uncertainties on all of the $\left|V_{u s}\right|$ measurements extracted from $\tau$ decays are limited by the experimental uncertainties. From Figure 3, it can be seen that the B-Factory measurements of $\left|V_{u s}\right|$ extracted from the ratio of $\frac{\mathcal{B}(\tau \rightarrow K v)}{\mathcal{B}(\tau \rightarrow \pi v)}$ and directly from $\mathcal{B}(\tau \rightarrow K v)$ are consistent with unitarity determined from[48]. These measurements are dominated by the BABAR measurement [5]. Interestingly, the value of $\left|V_{u s}\right|$ extracted using the FESR method has a deviation from unitarity of $3.4 \sigma$. With the recent upper limits on the unmeasured $\tau$ decay modes, the possibility of this deviation resulting from missing decay modes is becoming smaller. Moreover, although the measurements of the hadronic $\tau$ decays at BELLE and BABAR seem to be systematically lower than the previous world averages, the sum of the branching ratios is consistent with unity. The deviation in the FESR approach for $\left|V_{u s}\right|$ and the systematically low branching ratios could be related 


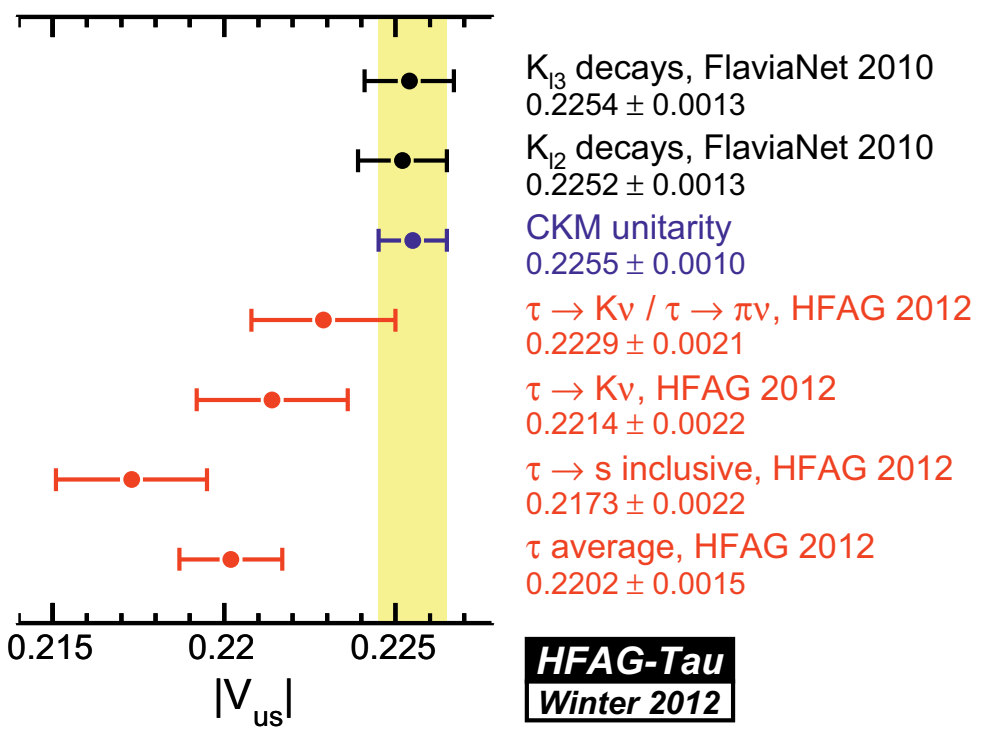

Figure 3. An update of $\left|V_{u s}\right|$ from the HFAG 2012 report[1] for the hadronic $\tau$ decays. The HFAG values of $\left|V_{u s}\right|$ are extracted using the average branching fractions from HFAG. The three upper values are from $K_{l 3}$ decays [49], $K_{l 2}$ decays [49] and the unitarity constraint [48].

to the difference in the definitions of the decay modes between the B-Factories and previous experiments especially given that the $\pi^{0}$ branching ratios tend not to be measured at BABAR and BELLE. On the theoretical side, the current weights used for the calculation of $\left|V_{u s}\right|$ have been shown to have potential problems with convergence [56, 65, 66] which are not included in the theoretical uncertainties. Unfortunately, the limited information on the strange spectral density functions prevents the use of more sophisticated weights which are not known to have problems with convergence. Therefore, it would be prudent to wait for future results before drawing conclusions on the meaning of the deviation.

\subsection{Charged Lepton Universality and $\tau$ Decays.}

There are numerous techniques for testing Charged Lepton Universality in $\tau$ decays. We will limit the techniques to those used at BABAR. The ratio of branching fractions $\mathcal{B}\left(\tau^{-} \rightarrow \mu^{-} \bar{v}_{\mu} \nu_{\tau}\right)$ and $\mathcal{B}\left(\tau^{-} \rightarrow e^{-} \bar{v}_{e} \nu_{\tau}\right)$ can be used to test Charged Lepton Universality between electrons and muons.

$$
\left(\frac{g_{\mu}}{g_{e}}\right)_{\tau}^{2}=\frac{\mathcal{B}\left(\tau^{-} \rightarrow \mu^{-} \bar{v}_{\mu} v_{\tau}\right)}{\mathcal{B}\left(\tau^{-} \rightarrow e^{-} \bar{v}_{e} v_{\tau}\right)} \frac{f\left(m_{e}^{2} / m_{\tau}^{2}\right)}{f\left(m_{\mu}^{2} / m_{\tau}^{2}\right)}
$$

where $f(x)=1-8 x+8 x^{3}-x^{4}-12 x^{2} \log x$, assuming that the neutrino masses are negligible [38]. Charged Lepton Universality between the $\tau$ and $\mu$ leptons can be tested with

$$
\left(\frac{g_{\tau}}{g_{\mu}}\right)_{h}^{2}=\frac{\mathcal{B}\left(\tau \rightarrow h v_{\tau}\right)}{\mathcal{B}\left(h \rightarrow \mu v_{\mu}\right)} \frac{2 m_{h} m_{\mu}^{2} \tau_{h}}{\left(1+\delta_{h}\right) m_{\tau}^{3} \tau_{\tau}}\left(\frac{1-m_{\mu}^{2} / m_{h}^{2}}{1-m_{h}^{2} / m_{\tau}^{2}}\right)^{2}
$$

where the radiative corrections are $\delta_{\pi}=(0.16 \pm 0.14) \%$ and $\delta_{K}=(0.90 \pm 0.22) \%[62,67,68]$. The world average mass, lifetime values and meson decay rates were taken from [69].

The analysis in [5], also used the leptonic $\tau$ decays and the $\tau^{-} \rightarrow h^{-} v_{\tau}$ to test Charged Lepton Universality. Figures 4 and 5 present a comparison of the BABAR Charged Lepton Universality measurements to previous experiments. The BABAR measurements were consistent with the assumption of Charge Lepton Universality. It has been suggested that the small deviations in the $g_{\tau} / g_{\mu}$ from comparing $\tau^{-} \rightarrow h^{-} v_{\tau}$ to $h^{-} \rightarrow \mu^{-} \overline{v_{\mu}}$ could be related to the radiative corrections used in the analysis $[62,67,68]$.

\section{Measurements of the Hadronic Spectra and QCD}

The $\tau$ lepton sits at a unique energy near the thresholds of perturbation and low energy QCD. At these energies, one can probe the rich and interesting phenomena of low energy QCD, for example Okubo-Zweig-Iizuka suppression[8], Wess-Zumino mechanism[71, 72] and the mixing between the axial vector states $K_{1}(1270)$ and $K_{1}(1400)$ [73, 74], as well as measure fundamental parameters of the Standard Model. Parameters such as the strong coupling constant, $\alpha_{s}[75-80], m_{s}$ and $\left|V_{u s}\right|[56,65,66]$ can be extracted using the spectral functions. While the BFactories have measured many of the $\tau$ branching ratios, only spectral function for eight of the $\tau$ decay modes have been measured[6, 7, 12, 81, 82]. These invariant mass distributions are essential both to understand the hadronic structure of $\tau$ decays and to improve the simulation of $\tau$ decays for the LHC and future experiments. Recently, Tauola has been updated using the results from $[6,82,83]$ to improve the modeling of $\tau^{-} \rightarrow \pi^{-} \pi^{0} \nu_{\tau}, \tau^{-} \rightarrow \pi^{-} \pi^{-} \pi^{+} \nu_{\tau}$ and 


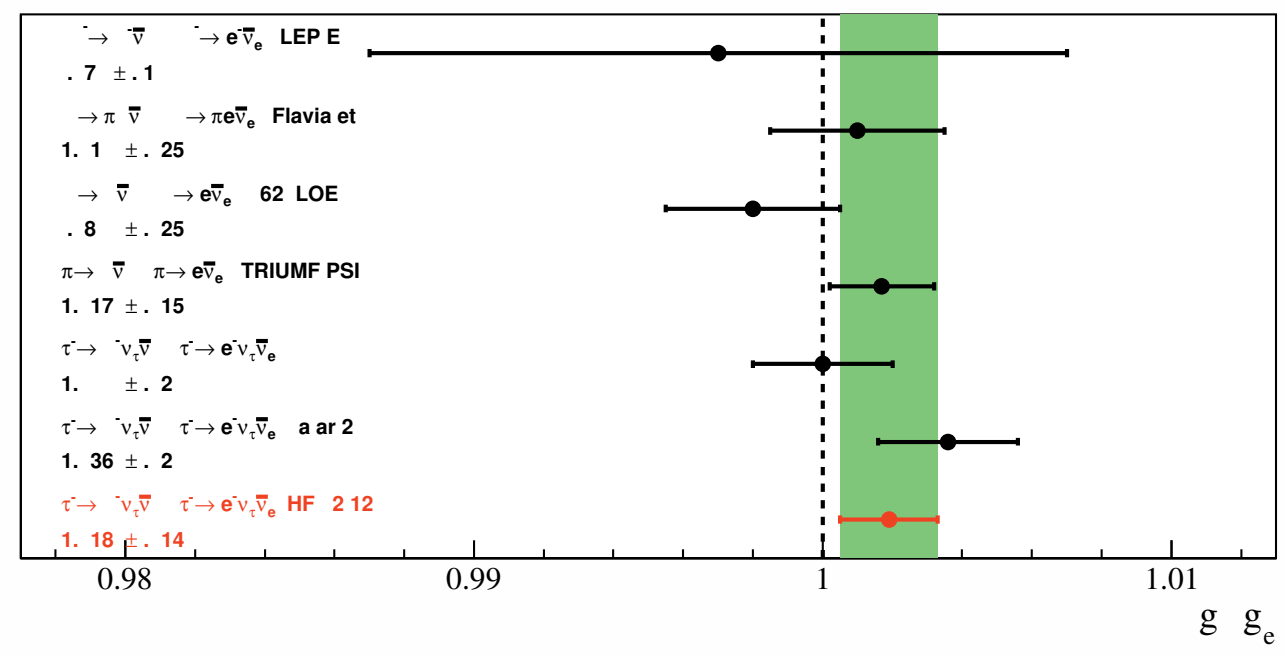

Figure 4. The current status of $g_{\mu} / g_{e}$ lepton universality measurements. The BABAR measurement is from [5], while the HFAG values are from[1]. The HFAG average [1] is the weighted average of previous $\tau$ results with the recent $B A B A R g_{\mu} / g_{e}$ measurement. The $\tau$ measurements from before BABAR are from [69]. The other measurements are taken from [1, 84].

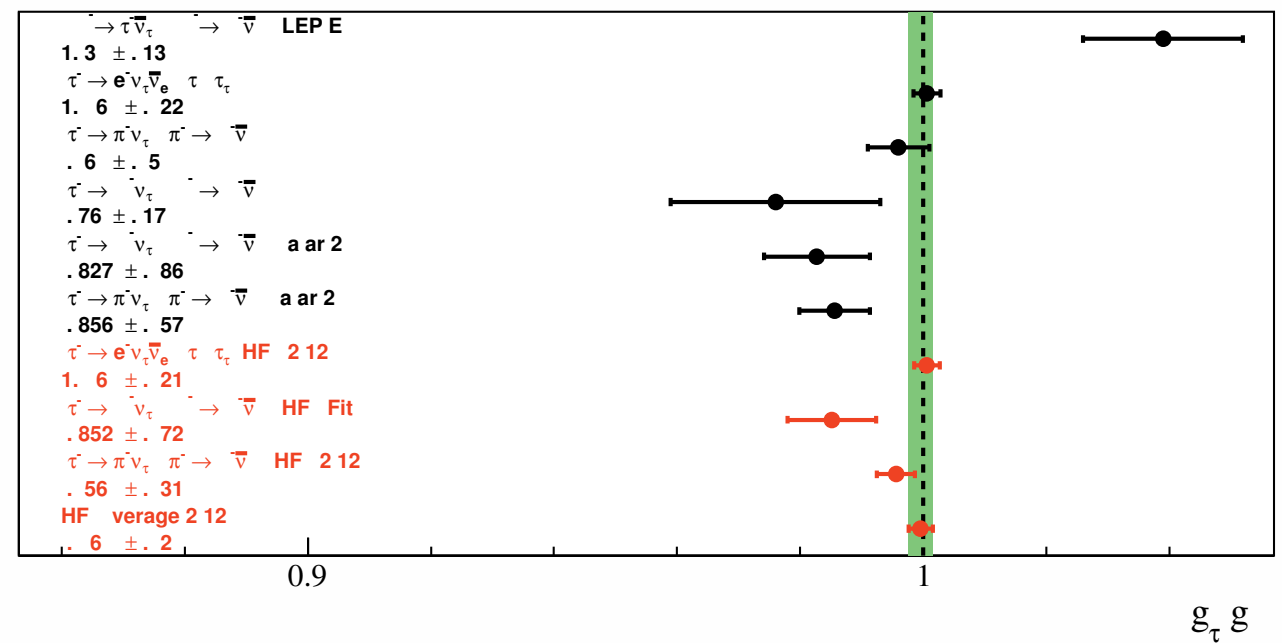

Figure 5. The current status of $g_{\tau} / g_{\mu}$ lepton universality measurements. The BABAR measurements are from [5], while the HFAG values are from[1]. The HFAG average [1] is the weighted average of previous $\tau$ results with the recent $B A B A R g_{\mu} / g_{e}$ measurement. The $\tau$ measurements from before BABAR are from [69]. The other measurements are taken from [1,84].

$\tau^{-} \rightarrow \pi^{-} \pi^{0} \pi^{0} v_{\tau}{ }^{1}$. Once a more complete strange and nonstrange hadronic spectra has been constructed, B-Factory data can be used to extract $\alpha_{s}, m_{s}$ and $\left|V_{u s}\right|$.

\section{Outlook}

With the large $e^{+} e^{-} \rightarrow \tau^{+} \tau^{-}$data sets collected by the B-Factory experiments and BES a substantial improve-

\footnotetext{
${ }^{1}$ The $\tau^{-} \rightarrow \pi^{-} \pi^{0} \pi^{0} v_{\tau}$ decay mode can be estimated using the $\tau^{-} \rightarrow$ $\pi^{-} \pi^{-} \pi^{+} v_{\tau}$ decay mode.
}

ment in our understanding of the properties of the $\tau$ has been made. The $\tau$ mass has been measured to an unprecedented precision while the electro-weak coupling between the first and second generations of quarks, $\left|V_{u s}\right|$, has been measured using complementary techniques to a level that is competitive with the kaon sector. Precision tests of Charged Lepton Universality have also been made. The hadronic spectra measured by the B-Factories are a significant improvement compared to previous experiments. These spectra allow one to probe the rich dynamics of low 
energy QCD in a clean environment as well as extract fundamental parameters. As it was shown in the Sections, 2 and $3 \tau$ decays also provide an ideal laboratory for searching for new physics. More specifically, the $\tau$ lepton is of particular interest due to the mass and flavour generation problem. Two mechanisms through which new physics can present itself at the B-Factories, are Lepton Flavour Violation and CP-Violations. Due to the extensive datasets from BABAR and BELLE, substantial progress has been made in these searches for physics beyond the Standard Model. In many cases the limits have been improved by several orders of magnitudes, drastically reducing the allowed parameter space for new physics models. With the next generation of B-Factories on the horizon, one can expect further progress in $\tau$ physics from the BELLE-II and BES-III experiments.

\section{References}

[1] Y. Amhis et al. [Heavy Flavor Averaging Group Collaboration], arXiv:1207.1158 [hep-ex].

[2] J. P. Lees [BaBar Collaboration], arXiv:1208.0376 [hep-ex].

[3] J. P. Lees et al. [BABAR Collaboration], arXiv:1209.2734 [hep-ex].

[4] S. Okubo, R. E. Marshak, E. C. G. Sudarshan, W. B. Teutsch and S. Weinberg, Phys. Rev. 112 (1958) 665.

[5] B. Aubert et al. [BABAR Collaboration], Phys. Rev. Lett. 105, 051602 (2010) [arXiv:0912.0242 [hepex]].

[6] M. Fujikawa et al. [Belle Collaboration], Phys. Rev. D 78, 072006 (2008) [arXiv:0805.3773 [hep-ex]].

[7] M. J. Lee et al. [Belle Collaboration], Phys. Rev. D 81, 113007 (2010) [arXiv:1001.0083 [hep-ex]].

[8] B. Aubert et al. [BABAR Collaboration], Phys. Rev. Lett. 100, 011801 (2008) [arXiv:0707.2981 [hepex]].

[9] K. Inami et al. [Belle Collaboration], Phys. Lett. B 672, 209 (2009) [arXiv:0811.0088 [hep-ex]].

[10] B. Aubert et al. [BaBar Collaboration], Phys. Rev. D 72 (2005) 072001 [hep-ex/0505004].

[11] B. Aubert et al. [BaBar Collaboration], Phys. Rev. D 77 (2008) 112002 [arXiv:0803.0772 [hep-ex]].

[12] S. Ryu [Belle Collaboration], arXiv:1302.4565 [hepex].

[13] B. Aubert et al. [BABAR Collaboration], Phys. Rev. D 76, 051104 (2007) [arXiv:0707.2922 [hep-ex]].

[14] B. Aubert et al. [BABAR Collaboration], Nucl. Phys. Proc. Suppl. 189, 193 (2009) [arXiv:0808.1121 [hepex]].

[15] S. Paramesvaran [BABAR Collaboration], [arXiv:0910.2884 [hep-ex]].

[16] P. del Amo Sanchez et al. [BABAR Collaboration], Phys. Rev. D 83, 032002 (2011) [arXiv:1011.3917 [hep-ex]].

[17] W. Liu, C. -X. Yue and J. Zhang, Eur. Phys. J. C 68 (2010) 197 [arXiv:0910.2514 [hep-ph]].
[18] W. -J. Li, Y. -Y. Fan, G. -W. Liu and L. -X. Lu, Int. J. Mod. Phys. A 25 (2010) 4827 [arXiv:1007.2894 [hep-ph]].

[19] Z. -H. Li, Y. Li and H. -X. Xu, Phys. Lett. B 677 (2009) 150 [arXiv:0901.3266 [hep-ph]].

[20] R. Benbrik and C. -H. Chen, Phys. Lett. B 672 (2009) 172 [arXiv:0807.2373 [hep-ph]].

[21] E. Arganda, M. J. Herrero and J. Portoles, JHEP 0806 (2008) 079 [arXiv:0803.2039 [hep-ph]].

[22] D. Black, T. Han, H. -J. He and M. Sher, Phys. Rev. D 66 (2002) 053002 [hep-ph/0206056].

[23] C. -H. Chen and C. -Q. Geng, Phys. Rev. D 74 (2006) 035010 [hep-ph/0605299].

[24] A. Ilakovac, Phys. Rev. D 62 (2000) 036010 [hep$\mathrm{ph} / 9910213]$.

[25] N. Combo, Phys. Rev. Lett. 10, 531 (1963).

[26] E. Christova, H. Eberl, W. Majerotto and S. Kraml, JHEP 0212 (2002) 021 [hep-ph/0211063].

[27] T. Ibrahim and P. Nath, Rev. Mod. Phys. 80 (2008) 577 [arXiv:0705.2008 [hep-ph]].

[28] S. Weinberg, Phys. Rev. Lett. 37 (1976) 657.

[29] Y. Grossman, Nucl. Phys. B 426 (1994) 355 [hep$\mathrm{ph} / 9401311]$.

[30] M. Bischofberger et al. [Belle Collaboration], Phys. Rev. Lett. 107 (2011) 131801 [arXiv:1101.0349 [hep-ex]].

[31] J. H. Kuhn and E. Mirkes, Phys. Lett. B 398 (1997) 407 [hep-ph/9609502].

[32] S. Y. Choi, K. Hagiwara and M. Tanabashi, Phys. Rev. D 52 (1995) 1614 [hep-ph/9412203].

[33] J. P. Lees et al. [BaBar Collaboration], Phys. Rev. D 85 (2012) 031102 [Erratum-ibid. D 85 (2012) 099904] [arXiv:1109.1527 [hep-ex]].

[34] S. Tisserant and T. N. Truong, Phys. Lett. B 115 (1982) 264.

[35] A. Bramon, S. Narison and A. Pich, Phys. Lett. B 196 (1987) 543.

[36] B. Aubert et al. [BaBar Collaboration], Phys. Rev. Lett. 103 (2009) 041802 [arXiv:0904.3080 [hepex]].

[37] S. Descotes-Genon, E. Kou and B. Moussallam, arXiv:1303.2879 [hep-ph].

[38] Y. -S. Tsai, Phys. Rev. D 4, 2821 (1971) [Erratumibid. D 13, 771 (1976)].

[39] J. Z. Bai et al. [BES Collaboration], Phys. Rev. D 53 (1996) 20.

[40] J. S. Schwinger, Phys. Rev. 91 (1953) 713.

[41] J. Schwinger, Phys. Rev. 93 (1954) 615.

[42] W. Pauli, Pergamon Press, Elmsford, NY (1955)

[43] G. L"uders, Kgl. Danske Videnskab Selskab., Mattfys. Medd. 28N5, (1954) 1.

[44] V. V. Anashin, V. M. Aulchenko, E. M. Baldin, A. K. Barladyan, A. Y. .Barnyakov, M. Y. .Barnyakov, S. E. Baru and I. V. Bedny et al., JETP Lett. 85 (2007) 347.

[45] Xiaohu Mo et al. [BESIII Collaboration], International Workshop on $e^{+} e^{-}$collisions from Phi to Psi 
(PHIPSI13).

[46] B. Aubert et al. [BaBar Collaboration], Phys. Rev. D 80 (2009) 092005 [arXiv:0909.3562 [hep-ex]].

[47] K. Abe et al. [Belle Collaboration], Phys. Rev. Lett. 99 (2007) 011801 [hep-ex/0608046].

[48] J. C. Hardy and I. S. Towner, Phys. Rev. C 79, 055502 (2009) [arXiv:0812.1202 [nucl-ex]].

[49] M. Antonelli, V. Cirigliano, G. Isidori, F. Mescia, M. Moulson, H. Neufeld, E. Passemar and M. Palutan et al., Eur. Phys. J. C 69, 399 (2010) [arXiv:1005.2323 [hep-ph]].

[50] M. Krawczyk and D. Temes, Eur. Phys. J. C 44, 435 (2005) [hep-ph/0410248].

[51] I. Dorsner, S. Fajfer, J. F. Kamenik and N. Kosnik, Phys. Lett. B 682, 67 (2009) [arXiv:0906.5585 [hep$\mathrm{ph}]$.

[52] W. Loinaz, N. Okamura, T. Takeuchi and L. C. R. Wijewardhana, Phys. Rev. D 67, 073012 (2003) [hep-ph/0210193].

[53] A. Czarnecki, W. J. Marciano and A. Sirlin, Phys. Rev. D 70, 093006 (2004) [hep-ph/0406324].

[54] W. J. Marciano, PoS KAON , 003 (2008).

[55] M. Antonelli, V. Cirigliano, A. Lusiani and E. Passemar, arXiv:1304.8134 [hep-ph].

[56] K. Maltman, C. E. Wolfe, S. Banerjee, J. M. Roney and I. Nugent, Int. J. Mod. Phys. A 23, 3191 (2008) [arXiv:0807.3195 [hep-ph]].

[57] C. T. H. Davies, C. McNeile, E. Follana, G. P. Lepage, H. Na and J. Shigemitsu, Phys. Rev. D 82, 114504 (2010) [arXiv:1008.4018 [hep-lat]].

[58] J. Laiho and R. S. Van de Water, arXiv:1112.4861 [hep-lat].

[59] A. Bazavov et al. [MILC Collaboration], PoS LATTICE 2010, 074 (2010) [arXiv:1012.0868 [hep-lat]].

[60] C. Kelly, arXiv:1201.0706 [hep-lat].

[61] S. Banerjee [BaBar Collaboration], arXiv:0811.1429 [hep-ex].

[62] W. J. Marciano and A. Sirlin, Lett. 71, 3629 (1993).

[63] W. J. Marciano, Phys. Rev. Lett. 93, 231803 (2004) [hep-ph/0402299].
[64] J. Erler, Rev. Mex. Fis. 50, 200 (2004) [hep$\mathrm{ph} / 0211345]$.

[65] J. Kambor and K. Maltman, Phys. Rev. D 62, 093023 (2000) [hep-ph/0005156].

[66] K. Maltman, Nucl. Phys. Proc. Suppl. 218, 146 (2011) [arXiv:1011.6391 [hep-ph]].

[67] R. Decker and M. Finkemeier, Nucl. Phys. Proc. Suppl. 40, 453 (1995) [hep-ph/9411316].

[68] R. Decker and M. Finkemeier, Phys. Lett. B 334, 199 (1994).

[69] W. M. Yao et al. [Particle Data Group Collaboration], J. Phys. G 33, 1 (2006).

[70] B. Aubert et al. [BaBar Collaboration], Nucl. Instrum. Meth. A 479, 1 (2002) [hep-ex/0105044].

[71] J. Wess and B. Zumino, Phys. Lett. B 37 (1971) 95.

[72] R. Decker and E. Mirkes, Phys. Rev. D 47 (1993) 4012 [hep-ph/9301203].

[73] G. Abbiendi et al. [OPAL Collaboration], Eur. Phys. J. C 13 (2000) 197 [hep-ex/9908013].

[74] D. M. Asner et al. [CLEO Collaboration], Phys. Rev. D 62 (2000) 072006 [hep-ex/0004002].

[75] F. Le Diberder [ALEPH Collaboration], In *Columbus 1992, Tau lepton physics* 191-201

[76] E. Braaten, S. Narison and A. Pich, Nucl. Phys. B 373 (1992) 581.

[77] S. Narison and A. Pich, Phys. Lett. B 211 (1988) 183.

[78] E. Braaten, Phys. Rev. Lett. 60 (1988) 1606.

[79] E. Braaten, Phys. Rev. D 39 (1989) 1458.

[80] K. Ackerstaff et al. [OPAL Collaboration], Eur. Phys. J. C 7 (1999) 571 [hep-ex/9808019].

[81] D. Epifanov et al. [Belle Collaboration], Phys. Lett. B 654, 65 (2007) [arXiv:0706.2231 [hep-ex]].

[82] I. M. Nugent, arXiv:1301.7105 [hep-ex].

[83] I. M. Nugent, T. Przedzinski, P. Roig, O. Shekhovtsova and Z. Was, Phys. Rev. D 88 (2013) 093012 [arXiv:1310.1053 [hep-ph]].

[84] K. Nakamura et al. [Particle Data Group Collaboration], J. Phys. G 37, 075021 (2010). 